EPJ Web of Conferences 41, 05022 (2013)

DOI: $10.1051 /$ epjconf/20134105022

C) Owned by the authors, published by EDP Sciences, 2013

\title{
Two-Dimensional Heterodyne-Detected VSFG Spectroscopy of Water Molecules at Charged Interfaces
}

\author{
S. Nihonyanagi, P. C. Singh, S. Yamaguchi, T. Tahara \\ Molecular Spectroscopy Laboratory, RIKEN, 2-1 Hirosawa, Wako, Saitama 351-0198, Japan
}

\begin{abstract}
Two-dimensional heterodyne-detected vibrational sum-frequency generation (2D-HD-VSFG) spectroscopy of water at interfaces has been realized for the first time. In the present study, 2D-HD-VSFG spectra were measured at a charged monolayer / isotopically diluted water interface. In contrast to the 2D-IR spectrum of bulk isotopically diluted water, the 2D-HDVSFG of the charged interface shows a narrower bleach band in the higher frequency region immediately after the photoexcitation. The results clearly show that the dynamics of the water at the charged interface is different from that in the bulk, reflecting the different environment where water molecules are located.
\end{abstract}

\section{Introduction}

Dynamics of liquid water have been one of the central subjects in the field of ultrafast spectroscopy. The water dynamics in the bulk has been intensively studied by various time-resolved (TR-) spectroscopies, and the ultrafast dynamics relating to the spectral diffusion, inhomogeneity, energy transfer etc. have been observed. Especially, two-dimensional infrared (2D-IR) spectroscopy has been playing a key role because it can provides detailed information about the pump \& probe frequencies dependency of the vibrational response and its temporal evolution with the change of the time delay [1]. On the other hand, the dynamics of interfacial water is much less understood compared with a wealth of knowledge about the bulk water dynamics. It is because an interfaceselective spectroscopic technique is needed to probe only interfacial water molecules in the presence of bulk water behind. The $2^{\text {nd }}$-order nonlinear spectroscopy, in particular vibrational sum frequency generation (VSFG), has intrinsic interface specificity and has been widely utilized to study steadystate properties of water at various interfaces [2]. Recently, TR-VSFG measurements (i.e., pumpprobe and 2D-VSFG) have been realized at aqueous interfaces [3-5]. However, conventional homodyne detection was employed in these TR-VSFG measurements, and hence only the absolute square of $2^{\text {nd }}$-order nonlinear susceptibility $\left(\left|\chi^{(2)}\right|^{2}\right)$ was measured. This absolute square nature of the homodyne detection causes substantial spectral deformation due to the interference between resonant peak(s) and nonresonant background, which prevent us from observing the true spectral response of the interfaces even in the steady-state measurement. This problem become much more serious in TR measurements because the transient signals are much weaker and the broad bleach signals are always accompanied by the hot band appearing with the opposite sign. Since these problems are absent in complex $\chi^{(2)}$ spectra [6], it is very crucial to realize heterodyne detection in TR-VSFG unrestricted use, distribution, and reproduction in any medium, provided the original work is properly cited. 
measurements to obtain time-resolved complex $\chi^{(2)}$ spectra. The time-resolved heterodyne-detected VSFG (TR-HD-VSFG)[7] [8] can directly provide the imaginary part of a pump-induced change in $\chi$ (2) $\left(\Delta \operatorname{Im} \chi^{(2)}\right)$, which makes interpretation as straightforward as the case of bulk transient absorption spectra that correspond to $\Delta \operatorname{Im} \chi^{(1)}\left(\chi^{(1)}\right.$; linear susceptibility). In the present paper, we extend our TR-HD-VSFG method [7] to 2D spectroscopy that is applicable to the liquid interface and report 2D-HD-VSFG spectra of water at an aqueous interface for the first time.

\section{Experimental}

The air/cetyltrimethylammonium bromide (CTAB) solution interface was chosen as a prototype of the positively charged aqueous interface. To remove inter- and intramolecular vibrational couplings, the isotopically diluted water $\left(\mathrm{HOD}-\mathrm{D}_{2} \mathrm{O}\right)\left(\mathrm{H}_{2} \mathrm{O}: \mathrm{HOD}: \mathrm{D}_{2} \mathrm{O}=1: 8: 16\right)$ was used as the aqueous phase. The optical layout is shown in Figure 1. The multiplex-detection with a broad

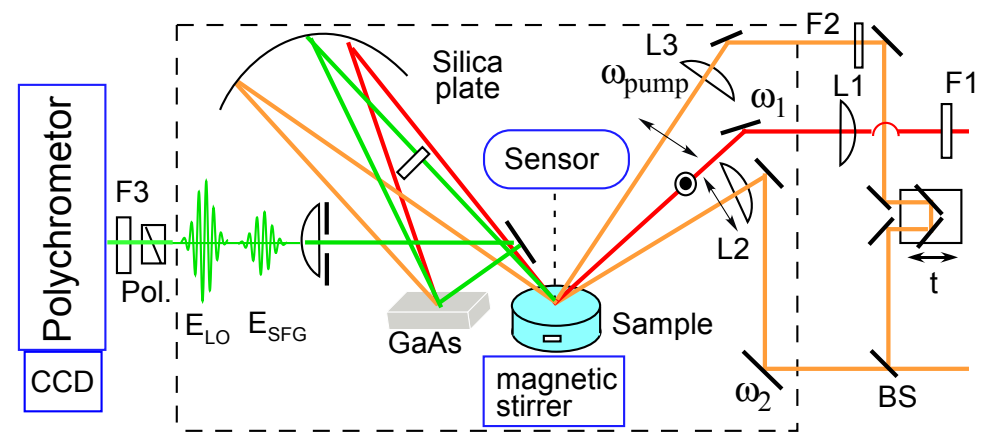

Fig.1 Optical layout of TR-HD-VSFG. F1: band pass filter centered at $795 \mathrm{~nm}$, bandwidth $1.5 \mathrm{~nm}$. F2: IR band pass filter. BS: 1:4 beam splitter. L1: silica lens, $\mathrm{L} 2$ and $\mathrm{L}$ : $\mathrm{CaF}_{2}$ lens.

band femtosecond IR-probe pulse provides $\operatorname{Im} \chi^{(2)}$ spectra in the entire $\mathrm{OH}$ stretch region with a high signal to noise ratio. For 2D-HD-VSFG measurements, $80 \%$ of IR light $\left(\omega_{2}\right)$ is split by a beam splitter (BS). The bandwidth of this IR is narrowed by a bandpass filter (F2: centered at $3300 \mathrm{~cm}^{-1}$, $3400 \mathrm{~cm}^{-1}$ or $3500 \mathrm{~cm}^{-1}$ ) down to ca. $100 \mathrm{~cm}^{-1}$, and it was used as the pump IR light $\left(\omega_{\text {pump }}\right)$. The $\omega_{\text {pump }}$ pulse first excited the $\mathrm{OH}$ stretch vibration of water, and the subsequent $\omega_{1}$ and $\omega_{2}$ pulses generated sum-frequency $\left(\omega_{\mathrm{SFG}}\right)$ pulse at the interface after a certain delay time. The electric field of the $\omega_{\mathrm{SFG}}$ light was measured through the interference with the local oscillator (LO) [6]. The $\Delta \operatorname{Im} \chi^{(2)}$ spectra obtained at different $\omega_{\text {pump }}$ were interpolated and combined to produce $2 \mathrm{D}$ plots. The instrumental response function measured as a three pulse cross-correlation was ca. $180 \mathrm{fs}$, allowing us to investigate the ultrafast dynamics of the interfacial water.

\section{Results and Discussion}

Figure 2 shows the 2D-HD-VSFG spectrum of the charged HOD- $\mathrm{D}_{2} \mathrm{O}$ interface obtained at a delay time (a) $\mathrm{t}=0$, (b) 0.1 and (c) $0.2 \mathrm{ps}$. In these 2D spectra, an upper positive band appears in the $\omega_{2}>$ $3250 \mathrm{~cm}^{-1}$ region and lower negative band appears in the $\omega_{2}<3250 \mathrm{~cm}^{-1}$ region. At the positively charged interface, the steady-state $\operatorname{Im} \chi^{(2)}$ spectrum shows a broad $\mathrm{OH}$ band having a negative sign, reflecting the net $\mathrm{H}$-down orientation of water molecules at the interface. Therefore, the positive signal in the 2D spectra corresponds to the bleaching of the negative ground-state $\mathrm{OH}$ band while the negative signal corresponds to the transient band attributed to the $v=1 \rightarrow 2$ hot band. In these 2D-HDVSFG spectra, the bleach band and the hot band can be unambiguously identified with their opposite sign. This is a sharp contrast to the homodyne TR-VSFG spectra where the bleach and hot band 

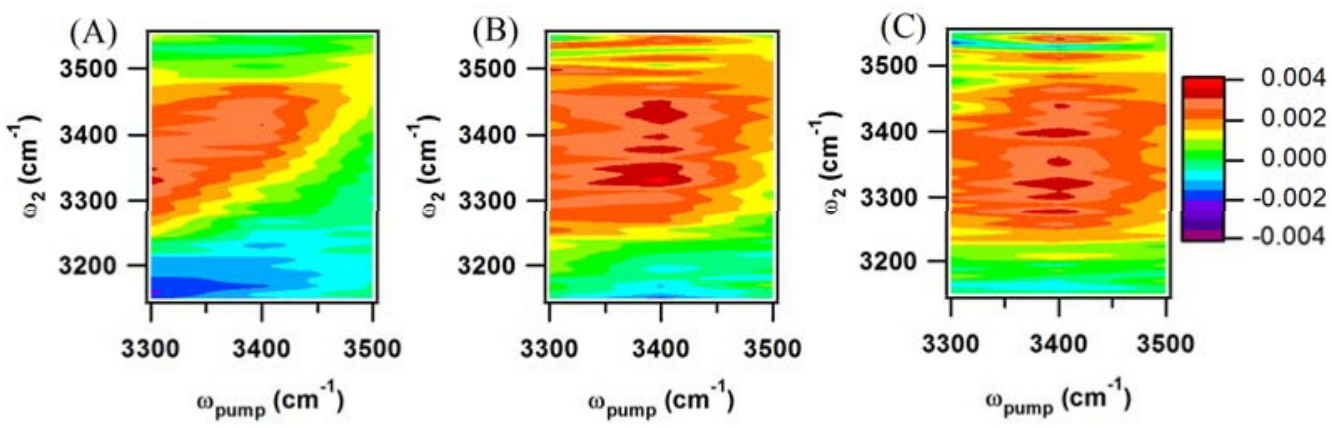

Fig.2 2D-HD-VSFG spectra of CTAB/HOD- $\mathrm{D}_{2} \mathrm{O}\left(\mathrm{H}_{2} \mathrm{O}: \mathrm{HOD}: \mathrm{D}_{2} \mathrm{O}=1: 8: 16\right)$ interface at $(A) \mathrm{t}=0$ ps, (B) 0.2 ps and (C) 0.4 ps.

appear as one broad band due to the square nature. This clearly demonstrates the necessity of the heterodyne detection to measure the true spectral response in time-resolved measurements.

The 2D-HD-VSFG spectrum at 0 ps (Figure 2A) clearly manifests frequency dependent line broadening for the spectral hole burnt by the pump pulse: With $\omega_{\text {pump }}$ at $3300 \mathrm{~cm}^{-1}$, the bandwidth of the spectral hole along the $\omega_{2}$ axis is as broad as $150 \mathrm{~cm}^{-1}$ whereas it is only $70 \mathrm{~cm}^{-1}$ with $\omega_{\text {pump }}$ at $3500 \mathrm{~cm}^{-1}$. At $0.2 \mathrm{ps}$ (Figure 2B), the spectral hole for $\omega_{\text {pump }}$ at $3300 \mathrm{~cm}^{-1}$ is broadened to $200 \mathrm{~cm}^{-1}$, indicating that the spectral diffusion is almost over at this delay time. For $\omega_{\text {pump }}$ at $3500 \mathrm{~cm}^{-1}$, on the other hand, the bandwidth is still $130 \mathrm{~cm}^{-1}$ at $0.2 \mathrm{ps}$, and thus the spectral diffusion is still ongoing. The 2D-HD-VSFG spectrum at 0.4 ps (Figure 2C) does not show any frequency dependent line broadening, which means that the spectral diffusion is completed for all $\omega_{\text {pump }}$. The present 2D-HDVSFG data clearly show that the spectral diffusion of the $\mathrm{OH}$ stretch at this charged interface is slower at higher $\omega_{\text {pump. }}$. This makes a striking contrast to the result of 2D-IR studies which showed that the spectral diffusion of the $\mathrm{OH}$ stretch band is faster at higher $\omega_{\text {pump }}$ in the bulk [1]. Based on MD simulations, it has been proposed that non hydrogen bond (NHB) water molecules, which give rise to the high frequency $\mathrm{OH}$ band, are intrinsically unstable in the bulk and their existence is only momentary, making the spectral diffusion faster [1]. The trend of the spectral diffusion observed for the charged HOD- $\mathrm{D}_{2} \mathrm{O}$ interface suggests the presence of a long-lived, weakly hydrogen bonded water at the interface.

\section{References}

1. J. J. Loparo, S. T. Roberts and A. Tokmakoff, J. Chem. Phys. 125, 12 (2006)

2. Y. R. Shen and V. Ostroverkhov, Chem. Rev. 106, 1140 (2006)

3. J. A. McGuire and Y. R. Shen, Science 313, 1945 (2006)

4. Z. Zhang, L. Piatkowski, H. J. Bakker and M. Bonn, Nat Chem 3, 888 (2011)

5. A. Eftekhari-Bafrooei and E. Borguet, J. Am. Chem. Soc. 131, 12034 (2009)

6. S. Nihonyanagi, S. Yamaguchi and T. Tahara, J. Chem. Phys. 130, 204704 (2009)

7. S. Nihonyanagi, P. C. Singh, S. Yamaguchi and T. Tahara, Bull. Chem. Soc. Jpn. 85, in press (2012)

8. W. Xiong, J. E. Laaser, R. D. Mehlenbacher and M. T. Zanni, Proc. Natl. Acad. Sci. U.S.A. 108, $20902(2011)$ 